\title{
Competencia comunicativa y adecuación de las pruebas oficiales de verificación. Un análisis sobre los DELE C2
}

Communicative competence and adequacy of the official verification tests. An analysis on the DELE C2

\author{
Palma Peña Jiménez \\ Universidad Rey Juan Carlos \\ España
}

\author{
Vicente Calvo Fernández \\ Universidad Rey Juan Carlos \\ España
}

José María Santos Rovira

Universidad de Lisboa Portugal

Palma Peña Jiménez: Departamento de CC. Educación, Lenguaje, Cultura y Artes, CC. Histórico-Jurídicas y Humanísticas, y Lenguas Modernas, Universidad Rey Juan Carlos, España. I E-mail: palma.pena@urjc.es José María Santos Rovira: Facultad de Letras, Universidad de Lisboa, Portugal.

| E-mail: jose.rovira@campus.ul.pt

Vicente Calvo Fernández: Departamento de CC. Educación, Lenguaje, Cultura y Artes, CC. Histórico-Jurídicas y Humanísticas, y Lenguas Modernas, Universidad Rey Juan Carlos, España.

I E-mail: vicente.calvo.fernandez@urjc.es 


\section{Resumen}

Nuestro objetivo es analizar si la prueba de comprensión lectora de los DELE C2 mide la competencia comunicativa del hablante. Partimos del principio de que cualquier alumno que desarrolla estudios universitarios en su lengua materna es un usuario competente, por lo que debe superar un examen que mida dicha competencia. Para verificar dicho supuesto, realizamos un estudio empírico de base cuantitativa con 176 alumnos españoles, a los que se les ha aplicado la prueba de comprensión lectora del DELE C2. Nuestra conclusión muestra que este examen no es adecuado, pues presenta un nivel de dificultad tan elevado que ni siquiera los universitarios españoles son capaces de superarlo.

Palabras clave: didáctica del español como lengua extranjera; MCER; competencia lectora; hablante nativo; usuario competente.

\section{Abstract}

Our aim is to check whether the reading comprehension test of the DELE C2 does actually measure communicative competence. Our starting point is that any student at university level in his/her mother tongue could be considered as a proficient user, so s/he must be able to pass a test oriented to measure reading competence. In order to check it, we conducted a case study on 176 Spanish students, to whom we have given the reading comprehension test of DELE C2. Our conclusion is that this exam is not suitable, as its level of difficulty is so high that even university native speakers cannot pass it.

Keywords: didactics of Spanish as a foreign language; CEFR; reading competence; native speaker; proficient user. 


\section{Introducción}

El DELE (Diploma de Español como Lengua Extranjera) es la titulación oficial reconocida por el Gobierno español, así como por la mayor parte de instituciones oficiales a nivel internacional, como certificativa de la competencia comunicativa en lengua española para hablantes no nativos y, en sus niveles $C$, acredita a estos como usuarios competentes. Basa sus presupuestos de una forma general en el Marco Común Europeo de Referencia para las Lenguas (en adelante MCER) (Consejo de Europa, 2002).

La pregunta que nos planteamos es la siguiente: ¿realmente los exámenes DELE sirven para medir la competencia comunicativa de un hablante? La cuestión no es baladí, puesto que la superación o no de estas pruebas puede tener consecuencias decisivas para la vida de cualquier hablante no nativo, tal y como mostraremos más adelante. La misma cuestión ha sido planteada en relación con todos los niveles marcados en el MCER, y también más allá del español, ya que

it is apparent that the CEFR levels have provided a convenient set of standards that have been enthusiastically adopted by government agencies in mandating targets for achievement or setting requirements for immigration programmes, often without any clear justification (Green, 2010: 15).

Es evidente que la comparación de niveles de competencia entre hablantes nativos vs. hablantes no nativos es compleja:

The very notion of a native speaker has, of course, been questioned and problematized, but there are obvious benefits to seeing how (similar) native speakers perform on the measures used by SLA researchers, even though we are conscious of the comparative fallacy (Alderson, 2010: 240).

Pero, aunque no exista un perfil único de hablante nativo, sino muchos, en función del nivel socioeducativo, en términos de competencia comunicativa es posible comparar los dos grupos de hablantes, nativos vs. no nativos, pues cabe medir las capacidades de comprensión y producción orales y escritas de unos y otros partiendo del supuesto de que cualquier hablante nativo tiene una elevada competencia comunicativa a nivel conversacional, de modo que es capaz de comprender y producir enunciados efectivos y adecuados en todos los contextos de su vida diaria. Y si nos centramos en las capacidades comunicativas de un hablante nativo de nivel universitario, podemos afirmar, además, que dicha competencia abarca todos los aspectos y contextos, con la única excepción de los contextos profesionales específicos.

De hecho, todo proceso de enseñanza/aprendizaje de lenguas extranjeras toma como referencia el habla de los nativos a la hora de decidir qué es correcto y usual, y qué es incorrecto o inusual: "The language content is thus built on idealized typifications of what native speakers may say and do in specified contexts" (Leung, 2005: 126). En efecto, todas las metodologías de enseñanza, así como todos los materiales didácticos creados con el propósito 
de enseñar una lengua extranjera a hablantes no nativos, basan sus temáticas, sus tareas, sus formas gramaticales, su léxico y todos sus contenidos en las producciones reales entre hablantes nativos. Por tanto, queda fuera de toda duda que son estos los modelos, de manera que cualquier aprendiente de una lengua extranjera cuenta con una referencia concreta y real sobre cómo debe producir sus enunciados orales o escritos.

Esta afirmación es compartida también por la literatura reciente. En concreto, De Haan y Van der Haagen (2013), en un estudio sobre la competencia escrita en inglés de los estudiantes holandeses, afirman que ellos toman como modelos de referencia las redacciones escritas por estudiantes nativos de nivel universitario, a la hora de evaluar las producciones escritas de sus propios alumnos: "We take the native undergraduate essays as a norm, beyond CEFR C2" (De Haan y Van der Haagen, 2013: 21). De la misma forma, Jokisch, Langenberg y Pintér (2014), en un trabajo sobre la competencia oral en lengua extranjera, confirman que la producción oral de cualquier hablante nativo es equivalente a la mejor producción oral que puede realizar el hablante no nativo de nivel C2: "We consider the highest L2 speaker level C2 equivalent to the mother tongue level of the L1 speakers" (Jokisch y otros, 2014: 797).

Por tanto, en nuestro trabajo hemos partido de la premisa de que un español nativo que cursa estudios universitarios debe de tener una avanzada competencia comunicativa, suficiente para poder describirle como usuario competente en su propia lengua materna, ya que cumple con lo marcado para tal nivel en el MCER:

Es capaz de comprender con facilidad prácticamente todo lo que oye o lee. Sabe reconstruir la información y los argumentos procedentes de diversas fuentes, ya sean en lengua hablada o escrita, y presentarlos de manera coherente y resumida. Puede expresarse espontáneamente, con gran fluidez y con un grado de precisión que le permite diferenciar pequeños matices de significado incluso en situaciones de mayor complejidad (Consejo de Europa, 2002: 26).

De este modo, a lo largo de nuestro estudio intentaremos verificar si la prueba de comprensión lectora de los exámenes DELE C2 es adecuada para evaluar la competencia comunicativa de un hablante y definirlo como usuario competente en español.

\section{En torno a la competencia comunicativa}

El primero en definir el concepto de competencia comunicativa fue Dell Hymes (1972), como reacción a la definición del concepto de competencia lingüística que unos años antes había planteado Noam Chomsky (1965). Según Hymes, la competencia comunicativa va mucho más allá de la competencia lingüística, se define como el conocimiento subyacente general y la habilidad para el uso adecuado de la lengua que posee el hablante-oyente, ya que la comunicación, además de ser formalmente posible (gramaticalmente correcta), debe ser también factible (según los medios de las personas que actúan), apropiada (según el contexto comunicativo) y realizable (basada en un comportamiento cultural real). Tener competencia comu- 
nicativa o ser competente no consiste solamente en conocer las reglas gramaticales de una lengua, concepto que tenían la mayoría de los lingüistas anteriores, sino que también incluye el conocimiento de las reglas de uso de la lengua, es decir, el uso adecuado al contexto y situación de comunicación.

Dentro de la didáctica de las lenguas, algunos autores han profundizado más en la definición de este concepto, añadiendo los componentes que deben enseñarse y evaluarse en la didáctica de las lenguas extranjeras para adquirir la deseada competencia comunicativa. Canale y Swain (1980) afirmaron que la competencia comunicativa consta de tres componentes fundamentales: el primero es la competencia gramatical, que engloba los conocimientos léxicos, sintácticos y morfológicos; en segundo lugar, la competencia sociocultural, que es el conocimiento de las reglas y normas de uso social y cultural; por último, la competencia estratégica está compuesta por las estrategias verbales y no verbales para compensar la falta de competencia lingüística. Posteriormente, Canale (1983) amplió los componentes a cuatro, diferenciando entre competencia sociocultural, que constituye las reglas socioculturales, y competencia discursiva, que es la capacidad de dar unidad gramatical y de significado a la comunicación. Algunos años después, Bachman (1990) incluyó cuatro componentes en su definición de competencia comunicativa y los distribuyó en dos bloques fundamentales: la competencia organizativa y la pragmática. La competencia organizativa integra la competencia textual, es decir, el conocimiento de los conectores para formar textos coherentes, y la gramatical, que engloba los conocimientos léxicos, sintácticos y morfológicos. La competencia pragmática estaría formada por la competencia léxica, que define como el conocimiento del significado de las palabras y la habilidad para hacer un uso figurativo de la lengua; la competencia funcional, que se define como el conocimiento de las relaciones entre enunciados e intenciones, y la competencia sociolingüística, que es el comportamiento lingüístico de una persona según el contexto cultural en el que se produce la comunicación. Posteriormente (Bachman y Palmer, 1996), añadiría un componente más a la competencia pragmática: el conocimiento funcional, al que definió como las intenciones comunicativas que muestran los hablantes. El trabajo de Celce-Murcia y otros (1995) siguió el modelo propuesto por Canale (1983), aunque dividiendo la competencia sociocultural en dos competencias diferenciadas: la propiamente sociocultural y la accional, a la que definieron como la capacidad para unir intenciones con formas lingüísticas.

La literatura que trató estas cuestiones (Celce-Murcia y otros, 1995; Hatch, 1992; Levinson, 1983; Olshtain y Cohen, 1991; Preston, 1989) deja muy claro que la competencia comunicativa de un hablante nativo es, en la práctica totalidad de los casos y contextos, superior a la de los no nativos, y subraya que gran parte de los actos comunicativos se realizan, en la vida real, en lo que denominan como "actos discursivos indirectos", los cuales obtienen una frecuencia de utilización tan elevada que los propios hablantes nativos ya no los consideran como indirectos, pero que siguen siendo fuente de innumerables problemas para los no nativos (Celce-Murcia y otros, 1995: 20). Más aún, la competencia comunicativa que posee el hablante 
nativo es tan amplia y abarca tantos contextos de utilización que, en la realidad, se convierte en inalcanzable para el hablante no nativo (Byram, 1997; Cook, 1999; Coperías, 2007).

A la luz de lo expuesto, sería paradójico poner en tela de juicio que un hablante nativo que se encuentre realizando estudios universitarios en su lengua materna no posea la competencia comunicativa a la que puede aspirar un hablante de español como lengua extranjera.

\section{EI MCER y los DELE}

Desde hace varias décadas ha existido la necesidad de establecer una serie de pruebas oficiales que sirvan para verificar la competencia lingüística, primero, comunicativa, posteriormente, de los hablantes de lenguas extranjeras. De esta forma, a lo largo del tiempo fueron surgiendo instituciones que comenzaron a crear sus propios modelos de exámenes y sus propias escalas de certificación. Con el establecimiento del Espacio Europeo de Educación Superior, se volvió necesaria la unificación de los dispares niveles existentes en cada lengua, por lo que en 2001 el Consejo de Europa presentó una escala de niveles de referencia que marcaba unos niveles comunes aplicables a todas las lenguas, el conocido como Marco Común Europeo de Referencia para las Lenguas (Consejo de Europa, 2002).

El establecimiento de una escala de niveles jerárquicos de competencia comunicativa en los que se marcan determinadas características específicas como obligatorias para ser reconocido como usuario básico, independiente o competente se vuelve extremadamente problemático, ya que hay muchos factores extralingüísticos que condicionan a los hablantes, más aún cuando el contexto en el que se trata de medir dicha competencia coloca al hablante fuera de su contexto diario, en el que, probablemente, se comunicaría con mayor soltura y eficacia. Además, el hecho de que los descriptores de determinadas competencias sean poco precisos complica mucho más la situación. Como ejemplo, en el nivel objeto de nuestro estudio, C2, el MCER describe que un hablante "sabe cómo sortear dificultades con tanta discreción que el interlocutor apenas se da cuenta" (Consejo de Europa, 2002: 39). La pregunta que cabe hacerse es si dicha formulación es la más adecuada para indicar cómo debe medirse la fluidez oral de un hablante.

La adecuación de los descriptores de niveles del MCER a la competencia comunicativa real de los hablantes es muy confusa, ya que no existe una relación clara entre el uso real de la lengua que un hablante puede hacer en su vida diaria y su clasificación como usuario básico, independiente o competente en función de aquellos. Tal y como dijimos anteriormente, entendemos que cualquier hablante nativo puede ser considerado como usuario competente; sin embargo, si leemos la totalidad de los descriptores de los niveles C, "cabe preguntarse cuántos hablantes nativos monolingües pueden hacer realmente todo esto" (Blake y Zyzik, 2016: 21).

Pero la falta de adecuación entre competencia comunicativa real y nivel de referencia alcanzado según lo exigido en el MCER tiene también la función inversa, es decir, que un hablan- 
te no nativo que en su vida real muestra una competencia comunicativa obviamente inferior a la de un nativo en múltiples contextos, como puede ser el caso de un alumno de una escuela internacional, podría perfectamente llegar a superar un examen de nivel C1 (North, 2014).

Esta falta de adecuación entre teoría y práctica, es decir, entre niveles marcados académicamente y uso real de la lengua, ha sido puesta de manifiesto en muchas ocasiones. Diversos estudios han querido establecer paralelismos entre las producciones de hablantes nativos y aprendientes de lenguas extranjeras, intentando comprobar si los requisitos exigidos por el referido MCER se corresponden adecuadamente con el supuesto nivel de competencia comunicativa que tendría el usuario que sea capaz de superarlos. Así, se define que un hablante de nivel B2, "utiliza con eficacia una variedad de conectores para marcar claramente las relaciones existentes entre las diferentes ideas" (Consejo de Europa, 2002: 38); sin embargo, recientes estudios demuestran la falsedad de esta premisa, puesto que los nativos, obviamente usuarios cuya competencia comunicativa es superior a dicho nivel B2, utilizan un mismo conector con el doble de frecuencia que los no nativos (Carlsen, 2010). Otro artículo recientemente publicado mostró que el uso de metáforas en los textos escritos (exigido por el MCER a partir del nivel B2) es mucho más al to entre hablantes de inglés como L2 que entre los propios nativos (Littlemore, Krennmayr y Turner, 2014).

La mayor parte de las investigaciones realizadas para la verificación de la adecuación de los criterios de valoración del MCER han sido realizadas teniendo como objeto de estudio a hablantes de inglés. No obstante, en uno de los escasos trabajos en los que se investiga a hablantes de español, encontramos conclusiones similares. En concreto, el trabajo de Rodrigo (2009) pone en evidencia que el criterio del MCER por el que se espera que un hablante no nativo de nivel B2 posea "un amplio vocabulario activo de lectura" (Consejo de Europa, 2002: 71) es excesivamente vago y difícilmente puede servir para verificar su competencia lingüística, ya que un estudio de las habilidades de comprensión lectora realizado con varios grupos de universitarios, unos hablantes nativos de español y otros aprendientes de ELE, demostró que algunos de los nativos analizados tenían un vocabulario más limitado que otros no nativos (Rodrigo, 2009: 585).

La falta de adecuación de los parámetros exigidos no queda reducida al nivel escrito, sino que se encuentran también muchos ejemplos a nivel de producción oral. Uno de los aspectos más controvertidos radica en el uso de las pausas en el discurso. Según los artífices del MCER, un hablante que aspire a ser considerado como "usuario competente" no debe realizar más que breves pausas en su discurso, ya que lo contrario podría ser considerado como indicativo de falta de fluidez oral. Sin embargo, estudios recientes desmienten tal supuesto y muestran que los hablantes nativos producen más pausas en su discurso que los hablantes de L2 de niveles avanzados (Hilton, 2014).

La misma descripción de lo que se considera como un "usuario competente" carece de toda justificación, al menos a nivel real, a no ser que se pretenda imponer un listón 
tan sumamente elevado que se vuelva inalcanzable para la casi totalidad de los hablantes. Pero la mayor objeción que podemos hacer al MCER es su falta de adecuación a la realidad. Queda fuera de toda justificación, por obvio, que un hablante nativo de nivel universitario representa el prototipo de usuario competente en la lengua, capaz de desenvolverse con fluidez en la práctica totalidad de contextos posibles y reales, con la única excepción de los ambientes técnicos, para los cuales es necesaria una formación específica. No obstante, ateniéndonos a los descriptores establecidos para los niveles C, nos damos cuenta de que dejan fuera a muchos de estos hablantes nativos (Blake y Zyzik, 2016). Por ello, debemos considerar a priori que dichos niveles carecen de fundamentación, puesto que han sido creados "without any thought being given to whether these levels might be achievable or justified" (Alderson, 2007: 662).

Por todo ello, consideramos que los niveles establecidos por el MCER son en gran medida artificiales y no responden al uso real de la lengua, y "si el propio sistema de niveles no deja de ser un 'artificio' en sí mismo, más artificiales serían aún los límites que puedan establecer dentro de cada nivel” (Ezeiza, 2016). De esta forma, coincidimos con muchos autores (Alderson, 2007; Blake y Zyzik, 2016; Ezeiza, 2016; Hulstijn, 2007; North, 2007; Weir, 2005) en que el actual MCER

is not sufficiently comprehensive, coherent or transparent for uncritical use in language testing. First, the descriptor scales take insufficient account of how variation in terms of contextual parameters may affect performances by raising or lowering the actual difficulty level of carrying out the target 'Can-do' statement (Weir, 2005: 281).

En el contexto concreto del nivel que nos ocupa, C2, ha quedado demostrado que no ha sido adecuadamente calibrado (North, 2007), ya que, en su forma actual, solo una minoría de hablantes nativos sería capaz de alcanzarlo (Hulstijn, 2010). Así, nos preguntamos, ¿es posible exigir a un hablante de una lengua extranjera un dominio de la misma que apenas un escaso porcentaje de hablantes nativos posee?

La normativa legal que creó los exámenes DELE estableció que estos serían las pruebas oficiales para mostrar la competencia lingüística en lengua española de los hablantes no nativos, estableciendo varios parámetros para determinar quién puede ser considerado como hablante nativo y, por extensión, quedar exento de tener que demostrar su competencia en esa lengua. Una de las características que inhabilita para obtener un DELE, según el artículo 2 del Real Decreto que regula estos diplomas, es el hecho de que una persona "haya cursado en español la totalidad o una parte de la educación primaria o secundaria” (Gobierno de España, 2002: 39490). Este condicionante es la evidencia de que, a nivel legal, cualquier estudiante universitario español puede ser considerado como usuario competente, con una competencia comunicativa superior a la de cualquier hablante de español como lengua extranjera, incluso de nivel C2. 
Del mismo modo, los alumnos extranjeros que acceden al sistema educativo español tienen que estar en posesión del DELE C2 para quedar exentos de las pruebas de idiomas (de acuerdo con el artículo 4.2 del Real Decreto 264/2008, que modifica la legislación anterior), titulación no exigida al que acredita ser hablante nativo (Gobierno de España, 2008). Frente a este presupuesto, nos preguntamos si realmente los hablantes nativos, con los que compartirá aula dicho extranjero, serían capaces de superar la prueba que a él le exigen. La pregunta es retórica, pues los estudios realizados hasta la fecha demuestran que

the $\mathrm{C}_{1}$ and $\mathrm{C}_{2}$ levels will generally not be attainable by L2 users with educational backgrounds other than higher education. In other words, they fail to consistently distinguish between L2 development and L2P (Hulstijn, 2011: 241).

En el caso concreto de los exámenes DELE, debemos puntualizar que

ni el Instituto Cervantes, el organismo principal responsable del sistema, ni la Universidad de Salamanca, la institución encargada de la elaboración y calificación de los exámenes, han mostrado hasta la fecha evidencia empírica alguna de que los exámenes o, mejor dicho, los resultados de los exámenes, sean fiables y válidos para los propósitos para los que fueron creados (Cárdenas, 2007: 393).

El motivo por el que no consideramos que estas pruebas sirvan para demostrar la competencia comunicativa de un hablante es su falta de fiabilidad, considerada como una de las cualidades que todo instrumento de evaluación debe poseer y que se ha definido como "la medida en que los resultados de un examen son estables, consistentes y libres de error" (Saville, 2003: 69).

La falta de fiabilidad de cualquier examen puntual para demostrar la capacidad comunicativa de un hablante ha quedado perfectamente expuesta en el trabajo de Berthold (2011), en el que él mismo alcanza una serie de resultados en exámenes de nivel de diferentes lenguas que en nada reflejan su auténtica competencia comunicativa. Basten los siguientes ejemplos: en inglés, lengua de la que es nativo y, para más inri, lingüista de profesión, es calificado en una de las pruebas realizadas como hablante de nivel intermedio; en francés, lengua en la que se considera muy competente (cursó estudios de postgrado en una universidad francesa), es calificado como hablante de nivel básico-avanzado; para mostrar más aún la falta de fiabilidad de dichos exámenes, realizó también varios en lenguas que nunca ha estudiado ni usado, como el español y el chino, en las que consigue obtener resultados que lo califican como usuario básico. Con respecto a los exámenes DELE, el trabajo de Cárdenas (2007) se orienta en la misma dirección y demuestra que dichas pruebas distan mucho de alcanzar los niveles de fiabilidad que poseen otras titulaciones de reconocimiento internacional, tales como TOEFL o IELTS, para llegar a la conclusión de que "Ios DELE tienen un nivel de fiabilidad extremadamente bajo" (Cárdenas, 2007: 403). 


\section{Estudio con estudiantes nativos españoles}

\subsection{Consideraciones previas}

El MCER establece una escala de seis niveles comunes de referencia de aprendizaje de una lengua. La tabla establece el siguiente modelo (Consejo de Europa, 2002):

I. Usuario básico: A1 y A2: Persona capaz de comunicarse en situaciones cotidianas, con vocabulario y gramática básica.

II. Usuario independiente: B1 y B2: Es capaz de desenvolverse en la mayor parte de las situaciones que pueden surgir durante un viaje por zonas donde se utiliza la lengua objeto de estudio y (B2) puede relacionarse con hablantes nativos con un grado suficiente de fluidez y naturalidad, de modo que la comunicación se realice sin esfuerzo por parte de los interlocutores.

III. Usuario competente: C1 y C2: reservando el "dominio operativo adecuado" para C1, nivel de competencia apropiado para tareas complejas de trabajo y estudio, y C2, que se ha denominado "Maestría", que implica una "competencia como la del hablante nativo o muy próxima".

Con la pretensión de comprobar en qué medida los exámenes DELE sirven para medir la competencia comunicativa en español, hemos llevado a cabo el siguiente estudio con alumnos universitarios de primer curso de grado de la Universidad Rey Juan Carlos de Madrid (España). La elección de la muestra responde a los siguientes criterios:

a) Nivel teórico del alumno universitario: C. Según el MCER, corresponde al nivel de usuario competente y a "personas con estudios académicos medios o superiores en su lengua materna”.

b) Un alumno extranjero debe presentar un nivel C2 para quedar exento de la prueba de idiomas al ingresar en el sistema educativo español (tal y como mostramos anteriormente); luego, se infiere que los alumnos nativos recién ingresados en la universidad deben superar las pruebas establecidas para dicho nivel.

c) La muestra está constituida por 176 estudiantes de cuatro grados diferentes. Este dato no parece a priori determinante, pero como el perfil del alumno puede presentar diferencias por su formación anterior y optativas diferentes en el bachillerato, la muestra se realiza a estudiantes de distintos grados, para que puedan estar representados perfiles heterogéneos.

d) Desde noviembre de 2011, el modelo de examen de DELE C2 presenta un formato con tres pruebas: la primera evalúa el uso de la lengua, y las otras dos, las destrezas. La primera prueba contiene seis actividades: tres sobre comprensión lectora y tres sobre comprensión auditiva. Además, se llevan a cabo otros ejercicios, que intentan medir la comprensión, interacción y mediación, tres sobre comprensión escritay destrezas integradas, más tres tareas de expresión oral. 


\subsection{Datos de la muestra y características de la prueba}

Los resultados del estudio, que constituye una primera fase de un proyecto más amplio sobre competencia comunicativa, deben confirmar si existen o no indicios que aconsejen realizar una investigación con una muestra mayor y con un control más preciso de variables.

Concretamente, esta prueba ha consistido en un test anónimo que se ha aplicado en el mes de septiembre de 2016 a estudiantes de primer curso de diferentes grados de la Universidad Rey Juan Carlos (URJC). La URJC es una institución universitaria pública con campus en distintas localidades del área metropolitana de la Comunidad de Madrid, España. Los estudiantes que constituyen la muestra están cursando grados de áreas de conocimiento diversas y en campus diferentes, como se explicará más adelante. Estos dos parámetros se controlan para comprobar si hay diferencias significativas, pero sobre la población muestral no se han tenido en cuenta en esta primera fase otros parámetros como la edad (más allá de que la mayoría de los estudiantes comienzan sus estudios con 18-19 años), el sexo, el tipo de centro del que proceden o el nivel socioeconómico.

Se han excluido de la prueba los estudiantes que no eran de nuevo ingreso en una universidad (es decir, aquellos que ya hubieran cursado alguna otra titulación universitaria, siquiera parcialmente) y aquellos estudiantes que no tenían el español como lengua materna. Es decir, se ha pretendido medir el nivel de comprensión lectora que presentan nativos de español al inicio de sus estudios universitarios.

El test que han realizado los sujetos es la parte primera (tres tareas - T1, T2 y T3 - de la prueba de uso de la lengua y comprensión lectora) de un examen escogido al azar de entre los propuestos en la convocatoria de noviembre de 2011 para la obtención del nivel C2 del DELE. Se incluye en el anexo una descripción de cada una de las tareas de que consta el examen.

Se ha aplicado el test durante una hora, que es el tiempo de que disponen los candidatos a obtener el título de DELE C2 para esta parte de la prueba. Los sujetos de la muestra solo sabían que estaban participando en una investigación (sin recibir más detalles sobre la misma) y que los resultados eran anónimos. En la realización del test no se ha controlado el orden de realización, que era libre (lo cual no ha permitido que se tuviera en cuenta la fatiga como variable). Tampoco se ha controlado la motivación de logro, puesto que los sujetos no obtenían ningún tipo de gratificación por realizar con éxito el test, pues la participación ha sido voluntaria, y la alternativa era disponer de una hora libre fuera del aula. De hecho, en varios grupos, algunos estudiantes escogieron esta opción y rehusaron realizar el examen.

La población muestral la han constituido estudiantes de los siguientes grados: 
a) 30 estudiantes del Grado en Ciencias Experimentales, que se cursa en la Escuela de Ciencias Experimentales y Tecnológicas del Campus de Móstoles, Madrid. La nota de corte para los estudiantes de esta titulación fue de 6.72 sobre 14 el curso de su ingreso, es decir, el 2016/17.

b) 60 estudiantes que están cursando el Grado en Protocolo, Organización de Eventos y Com. Corporativa o el Doble Grado Protocolo, Organización de Eventos y Com. Corporativa + RRII en la Facultad de Ciencias Jurídicas y Sociales (Campus de Madrid). Las notas de corte para el grado y el doble grado han sido, para el curso 2016/17, el de ingreso de esta cohorte, 8.66 y 12.41 , respectivamente.

c) 45 estudiantes del Grado en Educación Primaria y Educación Primaria con mención en Educación Musical, de la Facultad de Ciencias Jurídicas y Sociales del Campus de Móstoles, Madrid, cuya nota de corte ha sido 6.36 para el curso de ingreso 2016/17.

d) 41 estudiantes del Grado en Ingeniería en Sistemas Audiovisuales y Multimedia, de la Escuela Técnica Superior de Ingeniería de Telecomunicaciones (Campus de Fuenlabrada, Madrid), para el que la nota de corte el curso 2016/17 fue de 5.00.

Partiendo de la premisa de que los estudiantes que ingresan en una universidad pública española han adquirido la competencia lectora que se requiere para cursar ese tipo de estudios (pensar lo contrario supondría poner en duda la totalidad del sistema educativo español), cabe suponer que los sujetos que realizan la prueba no deben tener ningún problema para superarla con solvencia; de lo contrario, habría que cuestionar si la prueba, al menos en lo que se refiere al control de las habilidades relativas a la comprensión lectora, es adecuada para alumnos extranjeros cuya lengua materna no es el español, porque, en ese supuesto, estaría exigiendo un nivel de competencia lingüística o comunicativa muy superior al que presentan los nativos, tomando como normal el conocimiento y uso de la lengua propia de un estudiante que accede a la universidad.

\subsection{Resultados e interpretación}

En la tabla 1 se presentan los resultados de cada una de las tareas para los cuatro grupos de estudiantes. Los datos se ofrecen en una escala de 0 a 10 para facilitar la interpretación del rango en que se encuentra el grupo y con respecto a cada tarea. De manera global, la T2 y la T3 presentan una media significativamente baja. Por ejemplo, los resultados de la T3 quedan en la mayoría de los casos por debajo del 5 o del 50\% de aciertos: un 60\% de los alumnos de CC. Experimentales quedan en el rango inferior a 5; un 50\% en el caso del Grado en Protocolo; $57.77 \%$ en el caso de los alumnos que comienzan el Grado en Educación Primaria; y un 43.9\% en el caso de Ingeniería en Sistemas Audiovisuales y Multimedia. Pueden consultarse los promedios en la tabla 2. 


\section{TABLA 1}

Resultados por rango de calificación. Fuente: elaboración propia

\begin{tabular}{|c|c|c|c|c|c|c|c|c|c|}
\hline \multirow{6}{*}{$\begin{array}{l}\text { CC. } \\
\text { Experimentales } \\
30 \text { estudiantes }\end{array}$} & \multirow{2}{*}{ RANGO } & \multicolumn{2}{|c|}{ T1 } & \multicolumn{2}{|c|}{$\mathrm{T} 2$} & \multicolumn{2}{|c|}{ T3 } & \multicolumn{2}{|c|}{$\begin{array}{c}\text { TOTAL } \\
\text { ESTUDIANTES }\end{array}$} \\
\hline & & NÚM. & $\%$ & NÚM. & $\%$ & NÚM. & $\%$ & NÚM. & $\%$ \\
\hline & $9-10$ & 2 & 6.66 & 4 & 13.33 & 1 & 3.33 & 0 & 0 \\
\hline & $7-8.9$ & 11 & 36.66 & 3 & 10 & 1 & 3.33 & 6 & 20 \\
\hline & $5-6.9$ & 15 & 50 & 7 & 23.33 & 10 & 33.33 & 10 & 33.33 \\
\hline & $0-4.9$ & 2 & 6.66 & 16 & 53.33 & 18 & 60 & 14 & 46.66 \\
\hline Protocolo & & & & & & & & & \\
\hline 60 estudiantes & $9-10$ & 7 & 11.66 & 6 & 10 & 0 & 0 & 0 & 0 \\
\hline & $7-8.9$ & 28 & 46.66 & 6 & 10 & 13 & 21.66 & 15 & 25 \\
\hline & $5-6.9$ & 25 & 41.66 & 31 & 51.66 & 17 & 28.33 & 38 & 63.330 \\
\hline & $0-4.9$ & 0 & 0 & 17 & 28.33 & 30 & 50 & 7 & 11.66 \\
\hline
\end{tabular}

Ed. Primaria

45 estudiantes

\begin{tabular}{ccccccccc}
\hline $9-10$ & 2 & 4.44 & 2 & 4.44 & 0 & 0 & 0 & 0 \\
\hline $7-8.9$ & 18 & 40 & 0 & 0 & 3 & 6.66 & 5 & 11.11 \\
\hline $5-6.9$ & 23 & 51.11 & 18 & 40 & 16 & 35.55 & 23 & 51.11 \\
\hline $0-4.9$ & 2 & 4.44 & 25 & 55.55 & 26 & 57.77 & 17 & 37.77 \\
\hline
\end{tabular}

Ingeniería SS.

Audiovisuales

41 estudiantes

\begin{tabular}{ccccccccc}
\hline $9-10$ & 4 & 9.75 & 3 & 7.31 & 0 & 0 & 0 & 0 \\
\hline $7-8.9$ & 10 & 24.39 & 2 & 4.87 & 3 & 7.31 & 6 & 14.63 \\
\hline $5-6.9$ & 24 & 58.53 & 20 & 48.78 & 20 & 48.78 & 27 & 65.85 \\
\hline $0-4.9$ & 3 & 7.31 & 16 & 39.02 & 18 & 43.9 & 8 & 19.51 \\
\hline
\end{tabular}

Se ofrecen, además, los resultados para cada una de las tareas de que consta la prueba (figuras 1-3), y los resultados globales y sus porcentajes (figura 4): 


\section{FIGURA 1}

Resultados de la T1. Fuente: elaboración propia

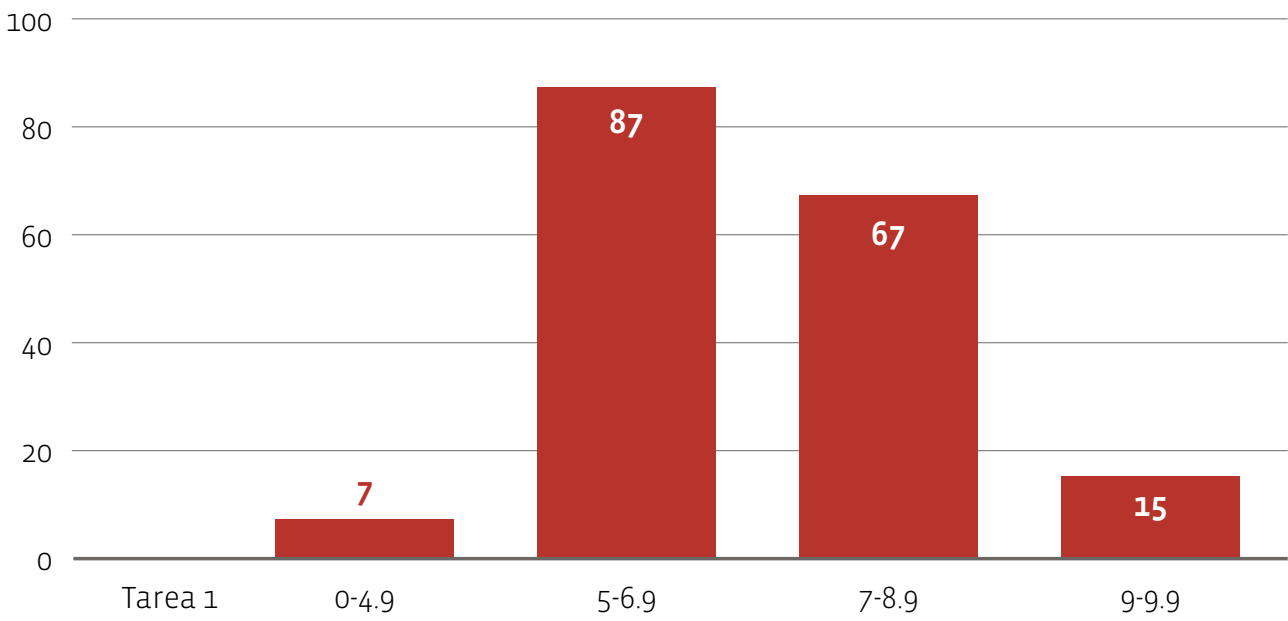

\section{FIGURA 2}

Resultados de la T2. Fuente: elaboración propia

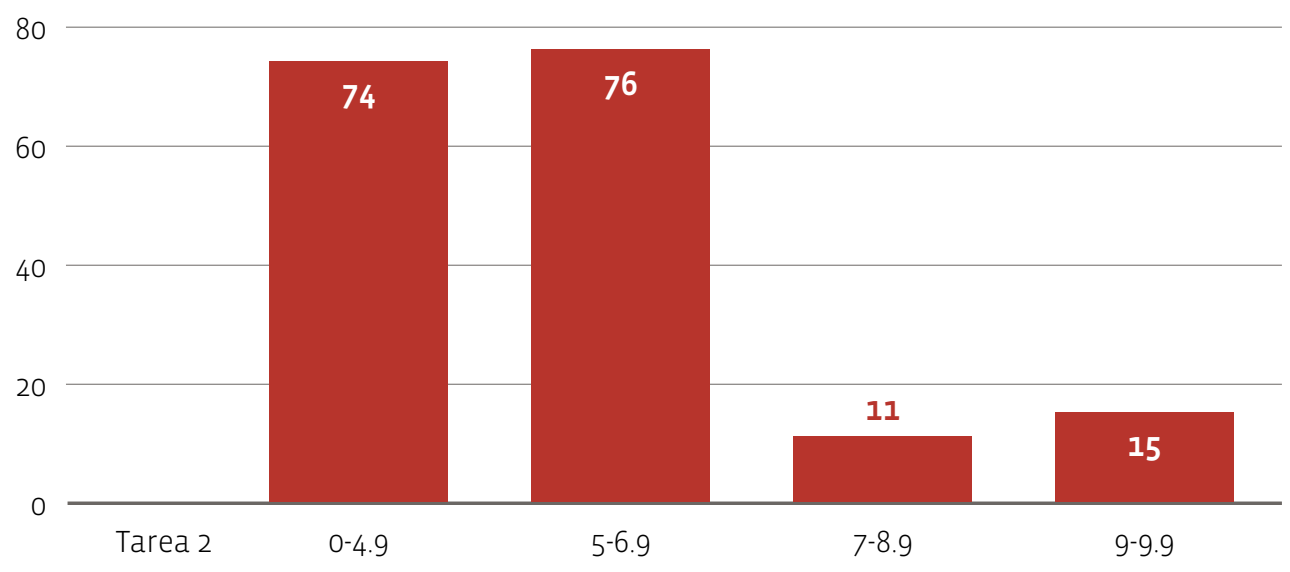

\section{FIGURA 3}

Resultados de la T3. Fuente: elaboración propia

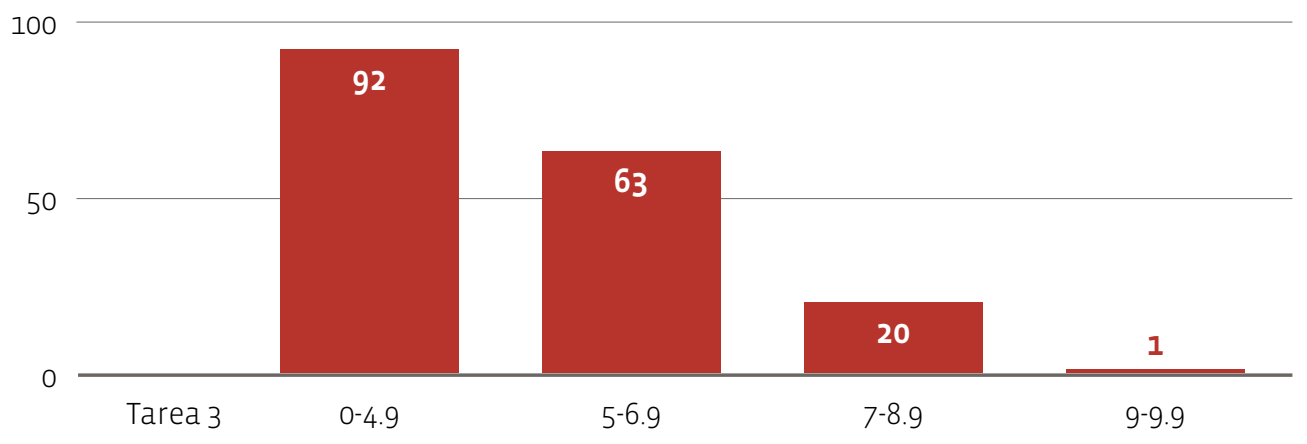




\section{FIGURA 4}

Resultados globales por rango de calificación. Fuente: elaboración propia

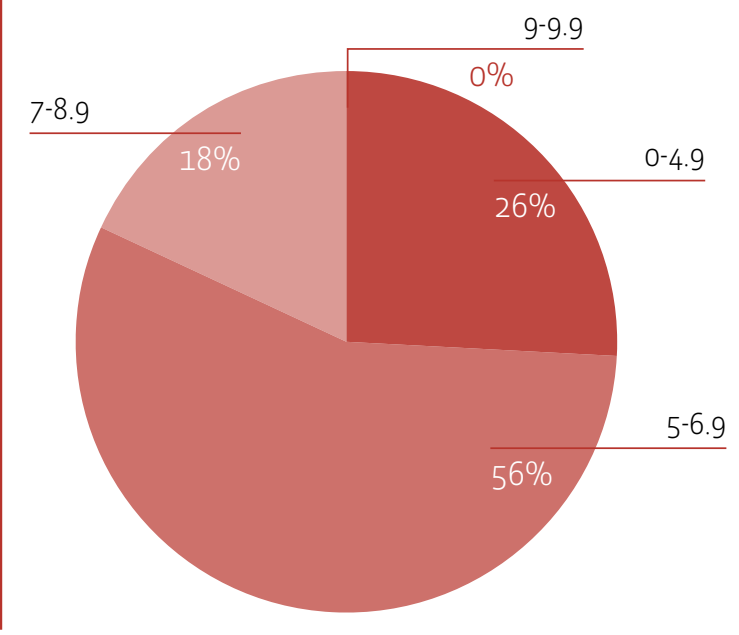

Como se ha dicho, el número total de estudiantes que participan en el estudio es de 176 , de los cuales 46 suspenden la prueba, es decir, no superan el 5 sobre 10. Estos representan un 26\%. 98 estudiantes quedan en el rango 5-6.9, lo que supone un 56\% de la muestra. En el rango 7-8.9 se sitúan 32 estudiantes, un 18\%. Ningún estudiante obtiene una calificación en el rango Sobresaliente (9-10).

A continuación, aportamos también los datos de promedio y desviación estándar de los resultados obtenidos por tareas:

\section{TABLA 2}

Estadísticos descriptivos. Fuente: elaboración propia

\begin{tabular}{lcc}
$\mathbf{N} 176$ & PROMEDIO & DESVIACIÓN ESTÁNDAR \\
Aciertos T1 (sobre 12) & 8.28 & 1.70 \\
\hline Aciertos T2 (sobre 6) & 2.99 & 1.51 \\
\hline Aciertos T3 (sobre 8) & 3.40 & 1.68 \\
\hline NOTA FINAL & 5.64 & 1.25 \\
\hline
\end{tabular}

En cuanto al índice de dificultad de estas pruebas, hemos de decir que solo se podría establecer estrictamente para cada una de las respuestas de la T1, porque en la T2 y T3 las respuestas incorrectas condicionaban el acierto o error en las restantes. Por ello, hemos calculado este indicador para cada tarea de manera global, considerándolas superadas si se resolvían correctamente el 50\% de los ítems de que constaban. De este modo, el índice de dificultad $p$ ha sido el siguiente: 


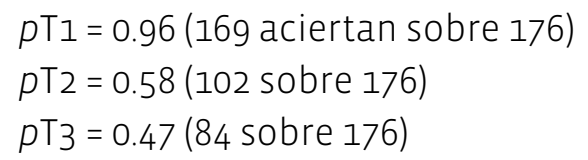

Por tanto, la T1 ha resultado fácil para los estudiantes nativos; en cambio, la T2 y la T3 presentan un nivel de dificultad medio-alto para los sujetos de la muestra, cuya lengua materna es, en todos los casos, el español.

Pero, además, el estudio de las correlaciones entre tareas arroja resultados relevantes. Hay diferencias cualitativas entre las habilidades que se exigen a los estudiantes en la T1 (precisión en la selección de léxico en un texto de tipo académico), en la T2 (comprensión lectora en el nivel de la microestructura textual: progresión temática, elementos conectores, etc.) y en la T3 (comprensión lectora en un nivel de macroestructura). Así lo sugieren las correlaciones halladas entre estas tareas, pues los coeficientes son <0.5 (ver tabla 3). Es decir, el acierto o error en una tarea es independiente del acierto o error en otra.

\section{TABLA 3}

Matriz de correlaciones. Fuente: elaboración propia

\begin{tabular}{lccc} 
VARIABLE & $\begin{array}{c}\text { ACIERTOS T1 } \\
\text { (SOBRE 12) }\end{array}$ & $\begin{array}{c}\text { ACIERTOS T2 } \\
\text { (SOBRE 6) }\end{array}$ & $\begin{array}{c}\text { ACIERTOS T3 } \\
\text { (SOBRE 8) }\end{array}$ \\
\hline $\begin{array}{l}\text { Aciertos T1 } \\
\text { (sobre 12) }\end{array}$ & 1 & & \\
\hline $\begin{array}{l}\text { Aciertos T2 } \\
\text { (sobre 6) }\end{array}$ & $\begin{array}{l}0.161^{*} \\
(0.033)\end{array}$ & 1 & 1 \\
\hline $\begin{array}{l}\text { Aciertos T3 } \\
\text { (sobre 8) }\end{array}$ & $\begin{array}{l}0.118 \\
(0.119)\end{array}$ & $\begin{array}{l}0.215^{\star *} \\
(0.004)\end{array}$ & 1 \\
\hline
\end{tabular}

* La correlación es significativa en el nivel 0.05 ( 2 colas)

** La correlación es significativa en el nivel 0.01 ( 2 colas)

Encuanto a lacapacidad discriminativa decada tarea, hemos calculado un índice de discriminación D dividiendo la muestra total N en dos grupos: uno formado por el $27 \%$ de los que obtienen el mejor resultado total y otro formado por el $27 \%$ de los que obtienen el peor resultado total. Por tareas:

$$
\begin{aligned}
& \mathrm{DT} 1=0.06 \\
& \mathrm{DT}_{2}=0.57 \\
& \mathrm{DT}_{3}=0.74
\end{aligned}
$$

Es decir, la T1 apenas discrimina (valor próximo a 0), pero la T2 y la T3 presentan buenos niveles de discriminación (valores más próximos a 1), pues quienes obtienen los mejores resultados globales son capaces, por regla general, de superarlas, pero fracasan en ellas los que obtienen los peores resultados de la prueba en su conjunto. 


\section{Conclusión}

El análisis de los resultados apunta a que la prueba de comprensión del examen DELE C2 tal vez no sea la adecuada para medir el nivel de competencia comunicativa real y, por tanto, para determinar que un estudiante no nativo sea considerado como usuario competente, puesto que un hablante nativo universitario tiene muchas dificultades para superarla, a pesar de que el diseño de la prueba, en cuanto a la variedad de tareas y el tipo de demandas que incluye, parece oportuno, a tenor de los indicadores.

Consideramos entonces que el problema principal de dichos exámenes radica en que parten de una perspectiva errónea con respecto a qué se debe medir con ellos. Si partimos de que el objetivo del proceso de aprendizaje de una lengua extranjera es adquirir la habilidad suficiente para poder realizar cualquier tipo de tarea en esa lengua, como un hablante nativo, toda prueba de nivel debe enfocarse de tal manera que lo que se mida sea, precisamente, lo que el hablante es capaz de hacer con dicha lengua, en lugar de prestar una atención excesiva a las singularidades lingüísticas (Alderson, 2010), que como acabamos de demostrar, ni siquiera los hablantes nativos universitarios son capaces de dominar.

Otro problema de base de este tipo de pruebas de dominio lingüístico radica en que están centradas exclusivamente en el estudiante universitario (Alderson, 2010), por lo que dejan fuera a todos aquellos hablantes que, pudiendo hacer un uso competente de la lengua en la mayor parte de las situaciones y contextos, no poseen ese nivel de estudios. El actual espectro de competencias exigidas en el MCER parece ignorar que la mayor parte de los hablantes de cualquier lengua no son capaces de realizar la mayor parte de las tareas exigidas en sus niveles C, pero no por ello podemos dejar de considerarlos como usuarios competentes en su lengua materna.

A modo de propuesta, consideramos que el Gobierno de España, a través del Ministerio que corresponda, debe adecuar la competencia lingüística exigida a los hablantes no nativos de español con el que poseen los nativos que entrarán en su mismo nivel educativo. De la misma forma, tanto la Universidad de Salamanca como el Instituto Cervantes deberían replantear las pruebas exigidas en los exámenes DELE, de forma que sirvan para medir la competencia comunicativa del aprendiente de español como lengua extranjera, tomando como base la competencia real que poseen los hablantes nativos, y no solo la de una minoría con excelentes habilidades lingüísticas.

\section{Bibliografía citada}

Alderson, J. Charles, 2007: "The CEFR and the need for more research", The Modern Language Journal 9 (4), 659-663. 
Alderson, J. Charles, 2010: "Language testing-informed SLA? SLA-informed language testing?" en Inge Bartning, Maisa Martin e Ineke Vedder (eds.): Communicative proficiency and linguistic development. Intersections between SLA and language testing research, European Second Language Association, 239-248.

Bachman, Lyle F., 1990: Fundamental considerations in language testing, Oxford: Oxford University Press.

Bachman, Lyle F., y Adrian S. Palmer, 1996: Language testing in practice, Oxford: Oxford University Press.

BerTHOLd, Michael, 2011: "Reliability of quick placement tests: how much faith can we place on quick paper or internet based placement tests", Australian Journal of Teacher Education 35 (6), 1-23.

BLAKE, Robert J., y Eve C. ZYZIK, 2016: El español y la lingüística aplicada, Georgetown: Georgetown University Press.

BrRam, Michael, 1997: Teaching and assessing intercultural communicative competence, Clevedon: Multilingual Matters.

Canale, Michael, 1983: "From communicative competence to communicative language pedagogy” en Jack C. Richards y Richard W. Schmidt (eds.): Language and communication, New York: Longman.

Canale, Michael, y Merrill Swain, 1980: "Theoretical bases of communicative approaches to second language teaching and testing", Applied Linguistics 1, 1-47.

CARDEnas, Abel, 2007: "La fiabilidad de la prueba de comprensión auditiva en los DELE: de la historia oficial a la realidad" en Las destrezas orales en la enseñanza del español L2-LE: XVII Congreso Internacional de la Asociación del Español como Lengua Extranjera (ASELE), Logroño: Universidad de La Rioja, 393-406.

CARLSEn, Cecilie, 2010: "Discourse connectives across CEFR-levels: a corpus based study" en Inge Bartning, Maisa Martin e Ineke Vedder (eds.): Communicative proficiency and linguistic development. Intersections between SLA and language testing research, European Second Language Association, 191-210.

Celce-Murcia, Marianne, Zoltán Dörnye y Sarah Thurrell, 1995: "Communicative competence: A pedagogically motivated model with content specifications", Issues in Applied Linguistics 6 (2), 5-35.

Chomsky, Noam, 1965: Aspects of the theory of syntax, Cambridge: MIT Press. 
Consejo de Europa, 2002: Marco Común Europeo de Referencia para las Lenguas: Aprendizaje, Enseñanza, Evaluación, Madrid: Ministerio de Educación, Cultura y Deporte, Instituto Cervantes y Grupo Anaya.

Cook, Vivian, 1999: "Going beyond the native speaker in language teaching", TESOL Quarterly $33(2), 185-209$.

CoperíAs, M. ${ }^{a}$ José, 2007: "Dealing with intercultural communicative competence in the foreign language classroom” en Eva Alcón y M. a Pilar SAfont (eds.): Intercultural language use and language learning, Dordrecht: Springer, 59-78.

De Haan, Pieter, y Monique van der Haagen, 2013: "Assessing the use of sophisticated EFL writing”, Dutch Journal of Applied Linguistics 2 (1), 16-27.

EzEIZA, Joseba, 2016: "El análisis de la complejidad lingüística en los niveles de competencia del MCER: retos y propuestas", Revista Nebrija de Lingüística Aplicada a la Enseñanza de Lenguas 20.

Gobierno de España, 2002: "Real Decreto 1137/2002, de 31 de octubre, por el que se regulan los diplomas de español como lengua extranjera (DELE)", Boletín Oficial del Estado 268, 39489-39481.

Gobierno de España, 2008: "Real Decreto 264/2008, de 22 de febrero, por el que se modifica el Real Decreto 1137/2002, de 31 de octubre, por el que se regulan los diplomas de español como lengua extranjera", Boletín Oficial del Estado 62, 14694-14696.

Green, Anthony, 2010: "Requirements for reference level descriptions for English", English Profile Journal $1(1)$, 1-19.

Hatch, Evelyn, 1992: Discourse and language education, Cambridge: Cambridge University Press.

HILton, Heather, 2014: "Oral fluency and spoken proficiency: considerations for research and testing” en Pascale Leclerce, Amanda Edmonds y Heather Hilton (eds.): Measuring L2 Proficiency. Perspectives from SLA, Bristol: Multilingual Matters, 27-53.

HuLstujn, Jan H., 2007: "The shaky ground beneath the CEFR: Quantitative and qualitative dimensions of language proficiency”, The Modern Language Journal 91 (4), 663-667.

Hulstuj, Jan H., 2010: "Linking L2 proficiency to L2 acquisition: Opportunities and challenges of profiling research" en Inge BartnIng, Maisa Martin e Ineke Vedder (eds.): Communicative proficiency and linguistic development. Intersections between SLA and language testing research, European Second Language Association, 233-238. 
Hulstijn, Jan H., 2011: "Language Proficiency in Native and Nonnative Speakers: An Agenda for Research and Suggestions for Second-Language Assessment", Language Assessment Quarterly 8 (3), 229-249.

Hymes, Dell, 1972: "On Communicative Competence” en John B. Pride y Janet Holmes (eds.): Sociolinguistics. Selected Readings, Harmondsworth: Penguin, 269-293.

Instituto Cervantes, 2011: Diplomas de español. Convocatoria de 2011. C2. Prueba 1: Uso de la lengua, comprensión de lectura y auditiva. Código de examen: 27 [https://examenes.cervantes. es/sites/default/files/dele_c2_2011_1.pdf, fecha de consulta: 16 de junio de 2017].

JokIsCh, Oliver, Tristan LANGenberg y Gábor PINTÉR, 2014: "Intonation-based classification of Ianguage proficiency using FDA" en Nick CampbelL, Dafydd Gibbon y Daniel HiRst (eds.): Speech Prosody 7, Dublin: Trinity College, 795-798.

Leung, Constant, 2005: "Convivial communication: recontextualizing communicative competence”, International Journal of Applied Linguistics 15 (2), 119-144.

Levinson, Stephen C., 1983: Pragmatics, Cambridge: Cambridge University Press.

LitTlemore, Jeannette, Tina Krennmayr y James Turner, 2014: "An investigation into metaphor use at different levels of second language writing", Applied Linguistics 35 (2), 117-144.

NoRTH, Brian, 2007: “The CEFR illustrative descriptor scales”, Modern Language Journal 91 (4), 656-659.

NoRTH, Brian, 2014: The CEFR in practice, Cambridge: Cambridge University Press.

OLSHTAIN, Elite, y Andrew D. Cohen, 1991: "Teaching speech act behavior to nonnative speakers" en Marianne Celce-Murcia (ed.): Teaching English as a second or foreign language, Boston: Heinle \& Heinle, 154-165.

Preston, Dennis R., 1989: Sociolinguistics and second language acquisition, Oxford: Blackwell Publishing Inc.

RodRIGo, Victoria, 2009: "Componente léxico y hábito de lectura en hablantes nativos y no nativos de español”, Hispania 92 (3), 580-592.

SAVILLE, Nick, 2003: "The process of test development and revision within UCLES-EFL" en Cyril J. WeIR (ed.): Continuity and innovation: revising the Cambridge Proficiency in English examination 1913-2002, Cambridge: Cambridge University Press, 57-116. 
WeIR, Cyril J., 2005: "Limitations of the Common European Framework for developing comparable examinations and tests", Language Testing 22 (3), 281-300.

\section{Anexo: descripción de la prueba}

El examen se encuentra disponible en la página web del Instituto Cervantes (2011).

La primera tarea consiste en completar doce palabras que faltan a lo largo de un texto eligiéndolas de entre tres posibles que se ofrecen para cada una de las demandas. Se requiere, pues, un conocimiento preciso del léxico, incluyendo los rasgos connotativos que permitan escoger un término y no otro en un contexto determinado. Se ofrece, a modo de ejemplo, un párrafo del texto, que es una adaptación de un escrito de Juan Bosch, y, a continuación, las opciones para completar las palabras de ese fragmento (Instituto Cervantes, 2011: 4-5):

Lo primero que debe 1 una persona que se inclina a escribir cuentos es la intensidad de su vocación. Nadie que no tenga vocación de cuentista puede llegar a escribir buenos cuentos. Lo segundo se refiere al género. ¿Qué es un cuento? La respuesta resulta tan difícil que a menudo ha sido ___ _ incluso por críticos excelentes, pero puede afirmarse que un cuento es el relato de un hecho que tiene indudable importancia. La importancia del hecho es desde luego relativa, mas debe ser indudable, convincente, para la 3 de los lectores. Si el suceso que forma el $4 \ldots$ __ del cuento carece de importancia, lo que se escribe puede ser un cuadro, una escena, una estampa, pero no es un cuento.
1. a) disipar
b) aclarar
c) interpelar
2. a) soslayada
b) inferida
c) propuesta
3. a) multitud
b) generalidad
c) amplitud
4. a) auge
b) meollo
c) rigor

En este caso, habría que elegir las opciones ab (“disipar”), 2a (“soslayada”), 3b (“generalidad”), 4b ("meollo").

En la segunda tarea, los aspirantes deben resolver un ejercicio que consiste en introducir en un texto científico divulgativo sobre la relación entre música y cerebro, que se presenta incompleto, los párrafos que faltan de un elenco que se facilita. Se trata, por tanto, de incluir cada parte en su lugar para que el resultado final resulte un discurso coherente y cohesionado. Los fragmentos que se facilitan son los siguientes (Instituto Cervantes, 2011: 7):

A. De aquí se puede concluir que la música es innata: nacemos dotados para apreciarla sin que nadie nos enseñe. La suposición es que las habilidades innatas son adaptaciones en el sentido evolucionista del término, capacidades que dan a los organismos mayores probabilidades de procrear y que, por lo tanto, van cundiendo en la población al paso de las generaciones hasta que solo quedan individuos con esas capacidades. 
B. Estos han evolucionado para indicarnos que hemos efectuado una acción que mejora nuestras probabilidades de vivir; por ejemplo, obtener alimentos llenos de energía para sobrellevar las épocas de "vacas flacas". Un pastel concentra estímulos placenteros que en cierta manera engañan al cerebro, haciéndole creer que hemos Ilevado a cabo una acción que promueve nuestra supervivencia.

C. Para extraerla e interpretarla el cerebro no actúa como una simple grabadora, sino que distribuye el estímulo sonoro a diversas regiones del cerebro donde se llevan a cabo los procesos de reconocimiento e interpretación.

D. Pero no todo el mundo les da importancia a estos asuntos, ni confía su imagen personal a su vestuario o a su biblioteca. Una expresión de identidad más común es la música que escuchamos.

E. Darwin, por su parte, pensaba que la música en los humanos surgió como herramienta para el cortejo, igual que la cola del pavorreal y el canto de muchas aves. Esta opinión es hoy minoritaria: si la música fuera de origen sexual, ¿ipor qué cumple tantas otras funciones y aparece en actividades tan diversas?

F. Para sustentar esta tesis, el autor señala que la música puede ser innata sin ser adaptativa, como otras formas de placer, por ejemplo, la gastronomía: el organismo solo exige nutrientes, sin requerir que estos vengan bien aderezados.

G. Charles Darwin lo expresó por primera vez en 1871, en su tratado sobre el origen de los humanos: "Puesto que ni la capacidad de disfrutar ni la de producir notas musicales tienen la menor utilidad para el hombre en sus hábitos cotidianos, hay que clasificarlas entre las facultades más misteriosas de las que está dotado". Se refiere a que las facultades musicales no nos sirven para defendernos de las fieras ni para cazar a nuestras presas; no calientan nuestro hogar, no nos ayudan a obtener agua ni cuidan nuestros cultivos.

Ejemplificamos con los dos primeros párrafos del texto (Instituto Cervantes, 2011: 6):

Hay quien expresa su identidad por medio de su atuendo y usa la ropa como si fuera una tarjeta de presentación. Otras personas se definen por lo que leen: se puede obtener mucha información acerca de ellas examinando el contenido de sus libreros. 13. La música nos gusta por diversas razones, pero sobre todo porque inspira emociones, desde la oleada de placer abstracto que nos pone la carne de gallina sin saber por qué, hasta la nostalgia del recuerdo que nos evoca. Tanto significado emocional le damos a la música que es fácil ponerse sentimental y no apreciar el enigma que entraña. Y es que, desde el punto de vista evolutivo, el origen de la música es un misterio. 14.

En el lugar 13. correspondería el fragmento D, y en el lugar 14., el fragmento G.

La tercera tarea, en la que se evalúa la comprensión lectora en un nivel de macroestructura, consiste en determinar el tema de una serie de resúmenes de ponencias tomadas de un 
congreso internacional sobre videojuegos, seleccionándolos de una lista dada. Los aspirantes deben leer detenidamente los textos y adjudicarles el tema que corresponde según su contenido. Hay seis textos (A-F) y ocho enunciados (19-26), por lo que algunos textos deben ser elegidos más de una vez. El enunciado de cada uno de los temas es el siguiente (Instituto Cervantes, 2011: 10):

19. Según esta ponencia existe un gran paralelismo entre la evolución del videojuego y el llamado "séptimo arte".
A)
B)
C)
D)
E)
F)

20. En esta ponencia se defiende un estudio del contexto con el que nos relacionamos a través de los videojuegos.
A)
B)
C)
D)
E)
F)

21. Se recuerdan en este trabajo los cambios que suponen algunos videojuegos en las relaciones interpersonales.
A)
B)
C)
D)
E)
F)

22. En esta ponencia se reflexiona sobre unos nuevos iconos que se utilizan en los videojuegos.
A)
B)
C)
D)
E)
F)

23. Según este trabajo los videojuegos se han visto influenciados por la revolución que suponen los nuevos medios de comunicarse y socializar Internet.
A)
B)
C)
D)
E)
F)

24. En este trabajo se dice que la vivencia de un lector o un espectador es radicalmente distinta a la de un usuario de videojuegos.
A)
B)
C)
D)
E)
F)

25. Esta ponencia analiza la adaptación de los temas narrativos tradicionales a nuevas formas de transmisión.
A)
B)
C)
D)
E)
F)

26. En este trabajo se afirma que los videojuegos pueden usarse para fines ajenos a lo puramente lúdico.
A)
B)
C)
D)
E)
F)

Transcribimos, a modo de ejemplo, el texto de una de las ponencias, la que corresponde a la opción A) (Instituto Cervantes, 2011: 8):

Otra lectura renovada gracias al videojuego. Federico Aldán: La comunidad artística está elaborando una gran variedad de modelos que renuevan el concepto de videojuego. Más allá de la consola y el monitor, el videojuego se extiende hacia el espacio urbano, tomando las tecnologías de la telecomunicación y la geolocalización como piedras angulares de las nuevas propuestas emergentes. El móvil -ahora microordenador - está presente en cada rincón del territorio; al vincularse a las redes sociales se extiende como un tejido computacional que articula todos los terminales de los 
países del primer mundo. Esta trama híbrida, real y virtual, es la nueva plataforma del juego. La interacción lúdica con nuestro entorno debería llevar implícito el análisis del mismo. El aquí y el allá, las zonas con y sin cobertura, lo urbano y lo rural, el control y la vigilancia, las fronteras reales de nuestra movilidad... El juego nos permite una lectura renovada del espacio físico y social, lectura que debería combinar la tecnología, las redes sociales, la geopolítica y por supuesto, la ecología.

En este caso, los temas arriba enunciados como 20 y 23 son los dos que se ajustan al contenido de este texto $A$ ). 